\title{
Editorial
}

\section{Ventricular remodelling after myocardial infarction}

Acute myocardial infarction can cause complex architectural changes in the ventricular myocardium that have an important bearing on ventricular function. In the past decade it has become evident that many patients with acute myocardial infarction undergo these complex changes and that these alterations affect outcome after acute myocardial infarction. Interest is growing in the evaluation of various interventions designed to reduce these changes and so improve left ventricular function and clinical outcome.

\section{Terminology, definitions, and time course}

We define ventricular remodelling as changes in shape and size of the left ventricle that can follow acute myocardial infarction. Infarct expansion and global ventricular dilatation are the processes that constitute ventricular remodelling. Infarct expansion, defined as disproportionate thinning and lengthening of the infarct zone resulting in regional distortion and dilatation of the cavity is seen within hours of the injury and is usually complete within three weeks. Global ventricular dilatation is defined as an increase in the overall size and distortion of the ventricular cavity resulting from expansion of the infarct zone and lengthening of the non-infarcted zone. Infarct expansion together with ventricular dilatation and the shape alterations seen up to about six weeks constitute the early remodelling phase and progressive ventricular dilatation seen from six weeks to about one year constitutes the late remodelling phase. ${ }^{1}$ During this late phase no important changes are seen in the scarred infarct zone.

\section{Infarct expansion}

Microscopically, infarct expansion is caused by myocyte slippage, which thins the infarct segment by reducing the number of cells across the myocardial wall. ${ }^{2}$ Grossly, the wall of the infarct zone shows thinning, lengthening, and regional cavity dilatation and bulging (figs 1 and 2 ).

\section{FACTORS ASSOCIATED WITH INFARCT EXPANSION}

Infarct expansion is characteristically seen in anteroapical transmural infarcts and is rare at other sites. Infarct

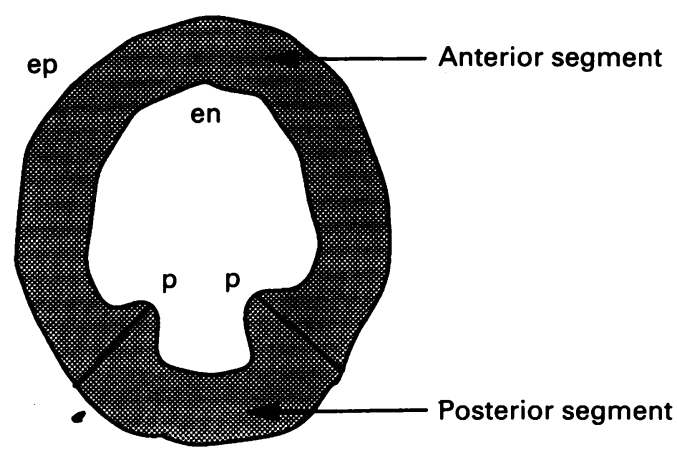

Figure 1 Diagram of a normal cross sectional short axis echocardiogram at mid papillary level showing normal myocardial thickness and cavity shape. p papillary muscles; en, endocardium, ep, epicardium. expansion occurs in approximately $40 \%$ of anteroapical transmural infarcts and $70 \%$ of such infarcts with fatal outcome in the first 30 days. ${ }^{34}$ Although a critical amount of myocardial necrosis, more than $10 \%$, is necessary to produce infarct expansion, the correlation between infarct size and the presence of expansion is weak. Other factors that promote expansion are increased loading conditions (both preload and afterload), and measures to reduce these have been shown to reduce expansion. ${ }^{56}$ Expansion is less likely where there is pre-existing hypertrophy, ${ }^{7}$ scar tissue from previous ischaemic episodes, and non-transmural infarcts (where a rim of viable epicardium splints the infarct area). Intramyocardial haemorrhage, associated with reperfusion therapy, and its subsequent organisation may alter the tensile strength of the infarct zone to make it more resistant to deforming forces. ${ }^{8}$ Administration of steroids and non-steroidal anti-inflammatory agents in the hours and days after myocardial necrosis promotes expansion by reducing the interstitial oedema and cellular infiltration that can provide mechanical support to the myocytes and prevent myocyte slippage. ${ }^{9-11}$

\section{CLINICAL CONSEQUENCES}

The increase in functional infarct size and the regional deformation of ventricular cavity shape have implications for left ventricular function and myocardial ischaemia in the non-infarcted, remote myocardium that may be perfused by a critically narrowed coronary vessel: therefore the presence of expansion is associated with reduced exercise tolerance caused by dyspnoea and angina. The presence of expansion is an independent risk factor for the development of left ventricular mural thrombus, frank ventricular aneurysm formation with its attendant complications, and cardiac rupture (both septal and free wall).

\section{Global ventricular dilatation}

The reduction in stroke volume and increased left ventricular filling pressure that result from the abrupt loss of segmental contractile function elicit two compensatory mechanisms initially. These are an increase in the contrac-

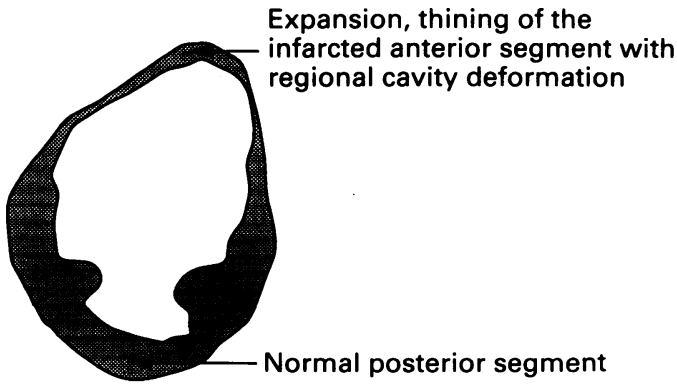

Figure 2 Diagram of cross sectional short axis echocardiogram at midpapillary muscle level in anterior myocardial infarction showing infarct expansion. 


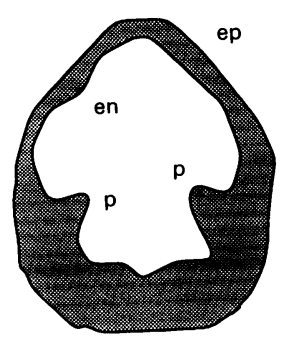

Day 1

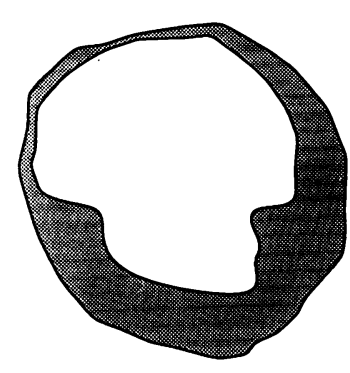

Day 10

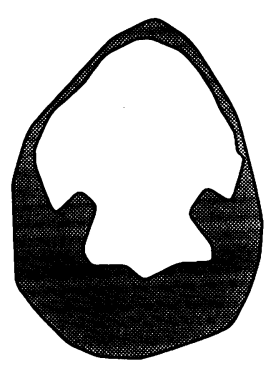

Day 3

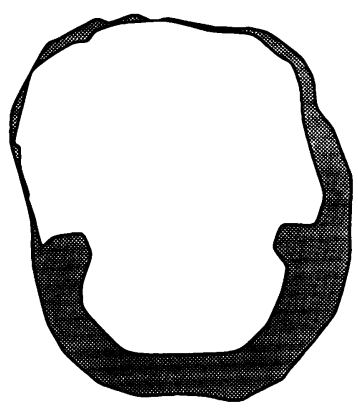

1 month
Figure 3 Diagrams of serial cross sectional short axis echocardiogram in anterior myocardial infarction showing infarct expansion (regional thinning and cavity deformation) and global ventricular dilatation (progressive global cavity dilatation and shape distortion). en, endocardium; ep, epicardium; $p$, papillary muscles.

tility of the viable myocardium stimulated by neurohormonal factors and a rise in end diastolic volume which through the operation of Frank-Starling mechanism serve to normalise filling pressure and restore stroke volume. These changes cause myocyte stretching, an increase in the length of the non-infarcted segment, and cavity dilatation. When the mass of the myocardial necrosis exceeds $20 \%$ of the total myocardial mass these two compensatory responses are inadequate to sustain optimal haemodynamic function and the third principal myocardial compensatory reserve, myocardial hypertrophy, is called upon. In patients with myocardial infarction this hypertrophy is the result of the addition of new sarcomeres in series which increases myocyte length and muscle mass without changing muscle thickness. This pattern of hypertrophy, called eccentric hypertrophy, is typical of volume overload states and results in chamber dilatation.

The disproportionate increase in cavity volume in relation to myocardial thickness produces a relative lack of decrease in the internal ventricular radius during ejection and this results in greater wall tension (Laplace's law) that is sustained during systole. This increase in systolic wall stress induces an increase in myocyte volume in the noninfarct zone that is typical of concentric hypertrophy. When these hypertrophic responses match the abnormal loading conditions haemodynamic function and cardiac size may stabilise but in large infarcts the hypertrophic response is inadequate both because of an insufficient increase in ventricular mass over cavity volume and insufficient response in the new capillary network to support the hypertrophy. Thus contractile function is impaired. ${ }^{12} \mathrm{~A}$ vicious circle is established in which abnormal loading conditions elicit an inadequate compensatory response that perpetuates the abnormal loading conditions and promotes progressive ventricular dilatation. Dilatation begets dilatation. In the early remodelling phase, infarct expansion together with changes in the non-infarct segment contribute to global ventricular dilatation whereas in the late remodelling phase ventricular dilatation is almost exclusively caused by changes in the non-infarct segment (fig 3).

\section{FACTORS ASSOCIATED WITH GLOBAL VENTRICULAR} DILATATION

Big infarcts, ${ }^{13}$ the presence of infarct expansion with the consequent effects of sustained abnormal loading conditions on the non-infarcted myocardium, and persistent occlusion of the infarct related coronary artery are the three important factors that promote progressive ventricular dilatation, ${ }^{1415}$ and the presence of clinically significant stenosis in the non-infarct related coronary artery has an important influence on the compensatory changes seen in the viable myocardium. ${ }^{16}$

\section{CLINICAL CONSEQUENCES}

The left ventricular volume is one of the most powerful predictors of mortality after myocardial infarction and progressive- ventricular dilatation is a precursor for the development of overt heart failure and is an important prognostic indicator of mortality after myocardial infarction. ${ }^{17}$

\section{Alterations in ventricular shape}

Alterations in ventricular shape and ventricular cavity geometry are important consequences of infarct expansion and global ventricular dilatation. Expansion of the necrotic myocardium and consequent regional shape distortion is a reversible elastic process, but after 24 hours plastic changes in the infarct zone result in permanent distortion though infarct expansion can still be reduced after this stage. Global ventricular dilatation results in a more spherical chamber: in general the degree of sphericity of the chamber seems to parallel increases in ventricular volume.

\section{CLINICAL CONSEQUENCES}

Preservation of dynamic and static ventricular geometry has implications for ventricular function ${ }^{18}$ and though the static geometry of the chamber seems to be related to the volume of the chamber it is tempting to suggest that the dynamic geometry of the dilated chamber and hence the function of the chamber may be independent of ventricular volume.

\section{Pharmacological interventions}

Reperfusion treatment with thrombolytic agents, early and late, is the most important pharmacological intervention that prevents infarct expansion in the early period after myocardial infarction. Early reperfusion therapy limits expansion by myocardial salvage while late reperfusion reduces expanion independently of myocardial salvage by limiting transmural damage and opening up collateral blood flow to the infarct zone thereby preserving a rim of viable epicardium. ${ }^{819}$ Infarct expansion may also be limited by unloading agents such as intravenous glyceryl trinitrate $^{20}$ and intravenous calcium channel blockers. ${ }^{21}$ It is conceivable that intravenous $\beta$ blockers, by reducing sympathetic activity and afterload, reduce the deforming forces acting on the infarct area and hence prevent expansion and cardiac rupture. ${ }^{22}$

Limitation of infarct size by reperfusion therapy and measures to reduce infarct expansion are the important interventions in the early hours after myocardial infarction that reduce global ventricular dilatation. Though persistent occlusion of the infarct related coronary artery and critical narrowing in the coronary vessels supplying the 
non-infarcted myocardium have an important bearing on global ventricular dilatation, the best method of achieving optimum coronary artery patency is still not clear.

There is evidence that ventricular unloading therapy in the first 24 hours with intravenous glyceryl trinitrate reverses the distortion of the cavity associated with infarct expansion and although reperfusion therapy also serves to preserve the regional shape the mechanism is one of myocardial salvage.

\section{ANGIOTENSIN CONVERTING ENZYME (ACE) INHIBITORS}

Recently the role of ACE inhibitors in the attenuation of ventricular remodelling has been increasingly explored and these are clearly an exciting class of agents. ACE inhibitors by reducing loading conditions and altering the neurohormonal response to myocardial infarction have been shown, experimentally, to reduce infarct expansion; some preliminary studies in patients treated with ACE inhibitors within few hours of infarction seem to confirm this. ${ }^{23-25}$ Certainly, we did not experience any important management problems when we used intravenous enalaprilat and oral enalapril within 24 hours of infarction in addition to streptokinase and intravenous glyceryl trinitrate.

There is now ample experimental and clinical evidence that the use of ACE inhibitors after infarct expansion has occurred or even in the late remodelling phase reduces global ventricular dilatation by optimising ventricular loading conditions and wall stress, ${ }^{2627}$ and possibly through alterations in the myofibre structure and protein and collagen content. ${ }^{28}$ Perhaps more exciting is the evidence that administration of ACE inhibitors a year after myocardial infarction resulted in partial reversal of left ventricular dysfunction. ${ }^{29}$

Experimental evidence indicates that ventricular unloading by use of ACE inhibitors in the first 24 hours reverses the regional shape distortion and that continued use of these agents in the recovery phase serves to preserve ventricular shape and internal geometry.

\section{Methods to study ventricular remodelling}

Methods available to study ventricular remodelling include cross sectional echocardiography, computed tomography, magnetic resonance imaging, radionuclide ventriculography, and contrast ventriculography. Cross sectional echocardiography, in view of its ease of performance of serial studies both in the early and follow up period, sophistication, and cost, provides the best practical way to study ventricular remodelling. The limitations of this technology are that in some patients the quality of the images may be unsatisfactory and direct visualisation of coronary anatomy impossible. The other non-invasive techniques of computed tomography and magnetic resonance imaging provide high quality tomographic images that are not limited by patient factors, although the high cost and the relative immobility of the equipment makes serial studies in the acute phase difficult. Alterations in ventricular volume and shape may be estimated by radioisotope ventriculography and contrast ventriculography and information about coronary anatomy may be obtained from coronary arteriography.

\section{Future directions}

Although currently available information suggests that the patient with a large, first, anteroapical myocardial infarction is at the greatest risk of ventricular remodelling, more information is required to assess the risk in different subgroups by means of a simple non-invasive test. Whereas reperfusion therapy with thrombolytic agents and unloading therapy by intravenous nitrates help to counteract remodelling the use of calcium channel blockers and $\beta$ blockers needs further clarification. The use of ACE inhibitors holds great promise and trials that are underway should further clarify the role of these agents. More research is also essential in defining the optimal combination of these various agents with reperfusion therapy and the timing of the start of therapy and duration of therapy that gives the best results.

MANI A VANNAN

Cardiac Department,

Kent and Canterbury Hospital,

Canterbury CT1 3NG. DERYCK J E TAYLOR

1 Erlebacher JA, Weiss JL, Weisfeldt ML, Bulkley BH. Early dilation of the infarcted segment in acute transmural myocardial infarction: Role of infarct expansion in acute left ventricular enlargement. J Am Coll Cardiol infarct expansi

2 Weisman HF, Bush DE, Mannisi JA, Weisfeldt ML, Healy B. Cellular mechanisms of myocardial infarct expansion. Circulation 1988;78: 186-201.

3 Pirolo JS, Hutchins GM, Moore GW. Infarct Expansion: Pathologic analysis of 204 patients with a single myocardial infarct. J Am Coll Cardiol 1986;7:349-54.

4 Weisman HF, Healy B. Myocardial infarct expansion, infarct extension and reinfarction: Pathophysiologic concepts. Prog Cardiovasc Dis 1987;30: 73-110.

5 Nolan SE, Mannisi JA, Bush DE, Healy B, Weisman HF. Increased afterload aggravates infarct expansion after myocardial infarction. $J A m$ Coll Cardiol 1988;12:1318-25.

6 Pierard LA, Albert A, Gilis F, Sprynger M, Carlier J, Kulbertus HE. Haemodynamic profile of patients with acute myocardial infarction at risk of infarct expansion. Am J Cardiol 1987;60:5-9.

7 Weisman HF, Udvarhelyi S, Bush DE, et al. Inducing hypertrophic shape changes in noninfarcted myocardium prevents progresssion of infarct expansion [abstract]. Clin Res 1984;32:216.

8 Hale SL, Kloner RA. Left ventricular topographical alterations in the completely healed rat infarct caused by early and late coronary artery reperfusion. Am Heart $J$ 1988;116:6:1508-13.

9 Mannisi JA, Weisman HF, Bush DE, Dudeck P, Healy B. Steroid administration after myocardial infarction promotes early infarct expansion. J Clin Invest 1987;79:1431-39.

10 Jugdutt BI. Delayed effects of early infarct-limiting therapies on healing after myocardial infarction. Circulation 1985;72:907-14.

11 Hammerman H, Schoen FJ, Braunwald E, Kloner RA. Drug induced expansion of infarct: Morphologic and functional correlations. Circulation 1984;69:611-7.

12 Anversa P, Beghi C, Kikkawa Y, Olivetti G. Myocardial infarction in rats. Infarct size, myocyte hypertrophy and capillary growth. Circulation Res 1986;58:26-37.

13 Kitamura S, Kay JH, Krohn BG, Magidson O, Dunne EF. Geometric and functional abnormalities of the left ventricle with a chronic localized noncontractile area. Am J Cardiol 1973;31:701-7.

14 Jeremy RW, Hackworthy RA, Bautovich G, Hutton BF, Harris PJ. Infarct artery perfusion and changes in left ventricular volume in the month after artery perfusion and changes in left ventricular volume in the m
acute myocardial infarction. J Am Coll Cardiol 1987;9:989-95.

15 Leung W-H, Lau C-P. Effects of severity of residual stenosis of the infarct related coronary artery on left ventricular remodelling and function after myocardial infarction [abstract]. Circulation 1991;84(suppl II):476.

$16 \mathrm{Kalil}$ R, Albuquerque C, Caramelli G, et al. Late function in the remote region following myocardial infarction: The importance of stenosis in the non-infarct related artery [abstract]. Circulation 1991;84(suppl II):476.

17 Jeremy RW, Allman KC, Bautovich G, Harris PJ. Patterns of left ventricular dilatation during the six months after myocardial infarction. $\mathrm{J} \mathrm{Am} \mathrm{Coll}$ Cardiol 1989;13:304-10.

18 Kass DA, Traill TA, Keating M, Altieri PI, Maughan WL. Abnormalities of dynamic ventricular shape change in patients with aortic and mitra valvular regurgitation: Assessment by fourier shape analysis and global geometric indexes. Circulation Res 1988;62:127-38.

19 Hochman JS, Choo H. Limitation of myocardial infarct expansion by reperfusion independent of myocardial salvage. Circulation 1987;75: 299-306.

20 Jugdutt BI, Warnica JW. Intravenous nitroglycerine therapy to limit myocardial infarct size, expansion and complications. Effect of timing, myocardial infarct size, expansion and complications.

21 Gottlieb SO, Gerstenblith G, Shapiro EP, et al. Nifedipine reduces early infarct expansion: results of a double-blind, randomised trial [abstract]. Circulation 1985;72(suppl III): 274 .

22 ISIS-1 Collaborative Group: Mechanisms for the early mortality reduction produced by beta blockade started early in acute myocardial infarction Lancet 1988;i:921-3.

23 Nabel EG, Topol EJ, Galeana A, et al. A randomized, double-blind, placebo controlled, pilot trial of combined early intravenous captopril and tPA therapy in acute myocardial infarction [abstract]. Circulation 1989;80 (suppl II): 112 .

24 Sharpe N, Smith H, Murphy J, Greaves S, Hart H, Gamble G. Early prevention of left ventricular dysfunction after myocardial infarction with angiotensin-converting-enzyme inhibition. Lancet 1991;337:872-6.

25 Pye M, Oldroyd KG, Ray SG, Christie J, et al. Effects of early captopri administration on left ventricular dilatation and function after acute myocardial infarction [abstract]. Circulation 1990;82 (suppl III):674.

26 Pfeffer JM, Pfeffer MA, Braunwald E. Influence of chroic captopril therapy on the infarcted left ventricle of the rat. Circulation Res 1985;57:84-95.

27 Pfeffer MA, Lamas GA, Vaughan DE, Parisi AF, Braunwald E. Effect of captopril on progressive ventricular dilatation after anterior myocardial captopril on progressive ventricular dilata

28 Michel JB, Lattion AL, Salzman JL, et al. Hormonal and cardiac effects of converting enzyme inhibition in rat myocardial infarction. Clin Res 1988; 62:641-50.

29 Sharpe N, Smith H, Murphy J. Late reversal of left ventricular dysfunction following myocardial infarction [abstract]. Circulation 1989;80(suppl II): 253 . 